\title{
Epidemiological and Evolutionary Features of Myopia in Melanoderma Children in Abidjan (Cote-D'ivoire): A 100 Cases Study in the University Hospital of Cocody
}

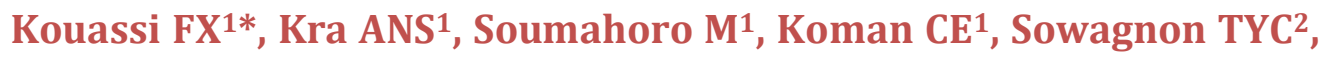 \\ Asso GE 1 and Koné $\mathrm{S}^{2}$ \\ 1Department of Ophtalmology, University Hospital of Cocody, Abidjan, Cote-d'Ivoire \\ ${ }^{2}$ Department of Ophthalmology, University Hospital of Yopougon, Abidjan, Côte-d'Ivoire
}

\section{Research Article}

Volume 3 Issue 4

Received Date: June 11, 2018

Published Date: July 06, 2018

*Corresponding author: François Xavier Kouassi, Certified Senior Lecturer in Ophthalmology, Head of the Department of Ophthalmology at University Hospital of Cocody, 22 BP 1368 Abidjan 22, Abidjan, Côte-d'ivoire, Tel: 00225 08-56-7966; Email: xavkouassi@yahoo.fr

\section{Abstract}

Introduction: Myopia is a spherical ametropia characterized by image formation in front of the retinal plane and responsible for a decrease in visual acuity from afar. The objectives of this study were to describe the clinical and evolutionary aspects of myopia in melanodermic children in Abidjan.

Materials and Methods: This is a retrospective and descriptive study that took place from 2010 to 2015 and focused on myopic subjects under 15 years of age who had 3 refractive studies, under cyclopentolate cycloplegia at $0.5 \%$, at regular 2-year intervals. All patients were equipped with corrective lenses.

Results: During the study period, 1647 children benefited from a refractive study. The prevalence of near sightedness was $19.30 \%$ (318 patients). One hundred children (200 eyes) with an average age of $8.47+/-2.08$ years were included in the study. There was a feminine predominance (69\%) with a sex ratio of 0.45 . Low myopia was predominant in the various examinations. The average progression of myopia after the first two years was $-0.45+/-0.48 \mathrm{D}$. An average worsening of $-0.35+/-0.45 \mathrm{D}$ between the $2 \mathrm{nd}$ and 4 th year was observed. The average increase in 4 years was $-0.80+/-$ $0.54 \mathrm{D}$.

Discussion: Myopia progressed during biological growth. This progression is influenced by genetic and environmental factors. We noted a slight progression of myopia in four years in melanodermic children in Abidjan.

Keywords: Refraction; Myopia; Evolution; Child; Melanoderma 


\section{Open Access Journal of Ophthalmology}

Abbreviations: WHO: World Health Organization; IAPB: International Agency for the Prevention of Blindness.

\section{Introduction}

Myopia is a spherical ametropia characterized by the formation of the image in front of the retina plane. The word myopic comes from two Greek words, "muein" which means to close and "ops" which means eye [1]. This refers to the attitude of myopic eyelids that closes the eyelids to achieve a pinhole effect that improves their distance vision. The optic nerve transmits a blurred image to the brain. The farther away the object is, the more blurred or nonexistent is the image. On the other hand, a nearsighted eye has good visual acuity. This condition is characterized by its progressive worsening during biological growth. It can cause visual impairment or blindness and is a real public health problem according to the World Health Organization (WHO). It is therefore rightly included as a target condition in the Global Vision 2020 Initiative of WHO and the International Agency for the Prevention of Blindness (IAPB) [2]. The objectives of this study were to describe the epidemiological and clinical features as well as the evolutionary particularities of this ametropia on a sample of melanodermic children in Abidjan in order to contribute to an improvement of its management in our context.

\section{Materials and Methods}

This is a retrospective and descriptive study, which ran from January 2010 to December 2015 at the Department of Ophthalmology in the University Hospital of Cocody and covered the files of myopic subjects under 15 years of age who, during that period, benefited from 3 studies of cycloplegic refraction under Cyclopentolate at $0.5 \%$, at regular 2 -year intervals. That is 3 refractive studies in 4 years. The protocol used consisted of instillation of three drops of Cyclopentolate at $0.5 \%$ spaced five minutes apart followed by an objective measurement of refraction at the Luneau $\AA$ refractometer (L65 E, France) 45 minutes after the first drop. A subjective refractive refraction refinement was carried out before prescription of the corrective lenses. We did not include in the study, the files of myopic children who did not have a refraction study at regular intervals of 2 years in 4 years, the refractions carried out according to another protocol and incomplete files. Myopia was distributed according to the diopteric classification into low ( $<3$ diopters), medium or moderate ( -3 to -6 diopters) and high (>-6 diopters) myopia [3]. The data were collected using a survey sheet. Data processing was carried out using Excel, Epi-data and Epi-info version 2000.

\section{Results}

\section{Headcount}

During the study period, 1647 ametropic children benefited from a refractive study according to the abovementioned protocol. The number of myopic children was 318 that is a prevalence of $19.30 \%$. Hyperopia had a prevalence of $65.33 \%$ (1076 patients) and astigmatism a prevalence of $63.99 \%$ (1054 patients). Only 100 cases that met the criteria for inclusion in the study were selected, representing $31.44 \%$ of nearsighted children and $6.07 \%$ of ametropic children in the service during the study period.

\section{Gender Distribution}

We noted a clear feminine predominance, with a sex ratio of 0.45: one male subject for two female subjects (Table 1).

\begin{tabular}{|c|c|c|}
\hline Gender & Effectives & Percentages \\
\hline Female & 69 & 69 \\
\hline Male & 31 & 31 \\
\hline Total & 100 & 100 \\
\hline
\end{tabular}

Table 1: Patient distribution by gender

\section{Patient Distribution by Age}

The average age was $8.47+/-2.08$ years with extremes ranging from 4 to 14 years. Children aged 6 to 10 accounted for $78 \%$ of the headcount (Table 2 ).

\begin{tabular}{|c|c|c|}
\hline Ages (years) & Headcount & Percentages \\
\hline 4 & 2 & 2 \\
\hline 5 & 5 & 5 \\
\hline 6 & 11 & 11 \\
\hline 7 & 16 & 16 \\
\hline 8 & 18 & 18 \\
\hline 9 & 19 & 19 \\
\hline 10 & 14 & 14 \\
\hline 11 & 8 & 8 \\
\hline 12 & 2 & 2 \\
\hline 13 & 3 & 3 \\
\hline 14 & 2 & 2 \\
\hline Total & 100 & 100 \\
\hline
\end{tabular}

Table 2: Patient Distribution by Age. 


\section{Initial Remote Visual Acuity without Correction}

Initial remote visual acuity without correction was less or equal to $5 / 10$ at $45 \%$ eye level (90 eyes) and greater than or equal to $8 / 10$ at $25 \%$ eye level (50 eyes). Sixty eyes (30\%) had a visual acuity far between $6 / 10$ and $7 / 10$.

\section{Prevalence of Different Types of Myopia at Initial Examination}

The classification of myopia as a function of diopter power at the beginning of the study gave the following proportions (Table 3):
Low myopia: 80\% (160 eyes)

Medium myopia: $15 \%$ (30 eyes)

Strong myopia: $5 \%$ (10 eyes)

\section{Evolution of Myopia at 2 Years}

The worsening of myopia after the first two years was observed in $89 \%$ of eyes, against $4 \%$ regression and $7 \%$ stability. The mean progression of myopia was $-0.45+/-$ 0.48 diopter (D) with extremes of -0.25 to $-2.25 \mathrm{D}$ (Figures $1 \& 2$, Tables $3 \& 4$ ).

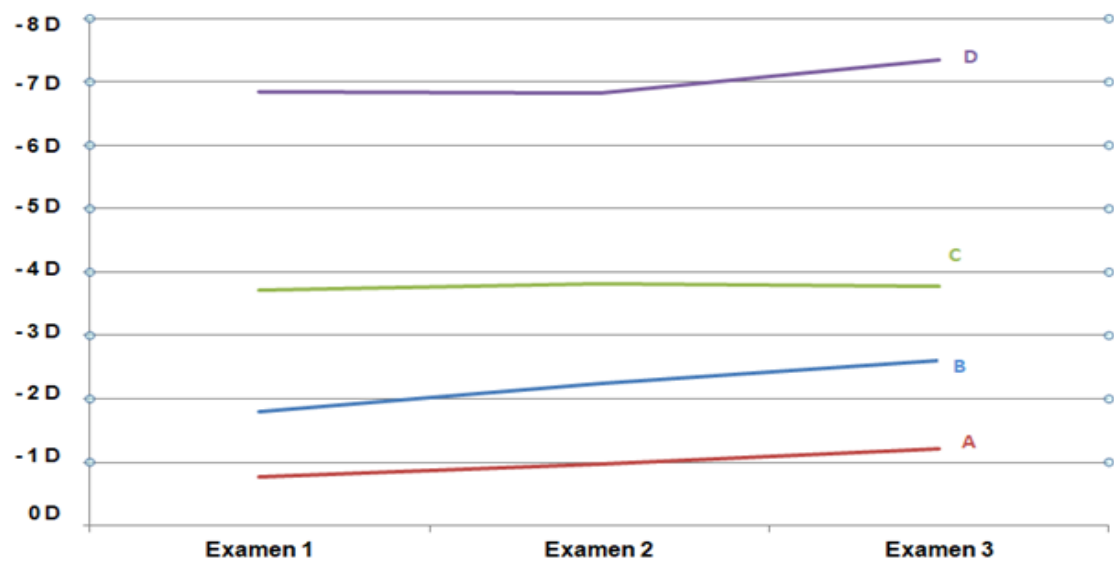

Figure 1: The average power progression curves of the myopia and its different stages. A: Average power of low myopia. B: Overall average power of myopia. C: Average power of moderate myopia. D: Average power of high myopia.

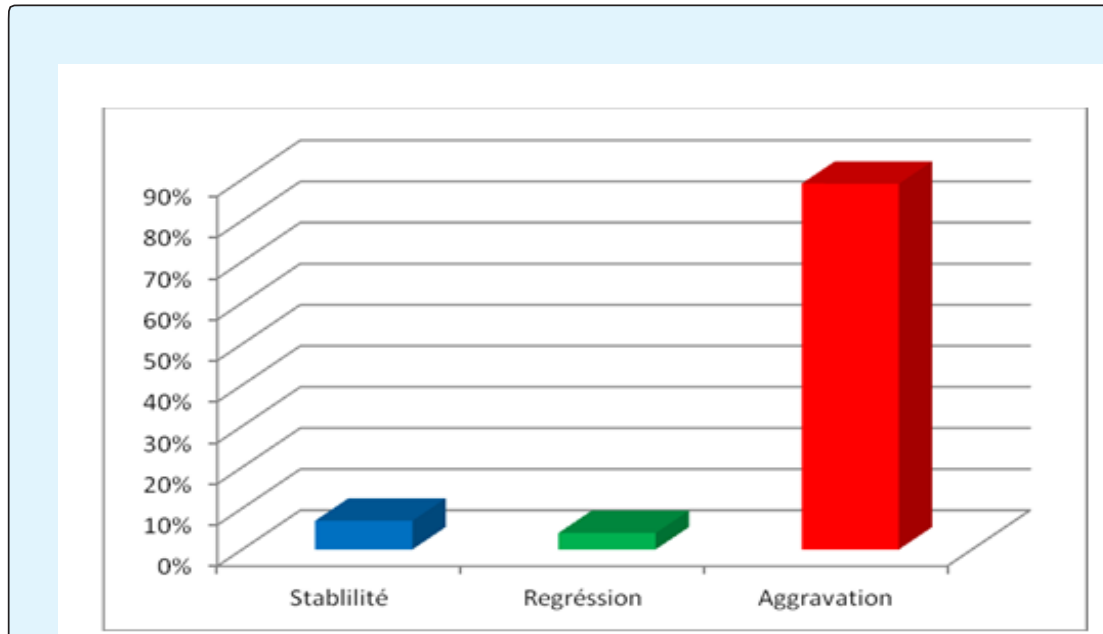

Figure 2: Evolution of myopia between initial examination and the 2nd year. 


\section{Open Access Journal of Ophthalmology}

\begin{tabular}{|c|c|c|c|}
\hline Periods & Types of myopia & Mean Power (D) & Frequencies (\%) \\
\hline \multirow{3}{*}{ Examination 1 } & Low & $-0,76+/-0,79$ & 80 \\
\cline { 2 - 4 } & Moderate & $-3,71+/-1,05$ & 15 \\
\cline { 2 - 4 } & Strong & $-6,85+/-0,51$ & 71 \\
\hline \multirow{3}{*}{ Examination 2 } & Low & $-0,97+/-0,79$ & 23 \\
\cline { 2 - 4 } & Moderate & $-3,81+/-1,08$ & 6 \\
\cline { 2 - 4 } & Strong & $-6,83+/-0,48$ & 70 \\
\hline \multirow{3}{*}{ Examen 3 } & Low & $-1,21+/-0,66$ & 25 \\
\cline { 2 - 4 } & Moderate & $-3,77+/-0,89$ & 5 \\
\cline { 2 - 4 } & Strong & $-7,35+/-0,49$ & \\
\hline
\end{tabular}

Table 3: Frequencies and Power Evolution of the Different Types of Myopia in 4 Years.

\begin{tabular}{|c|c|c|c|c|c|c|}
\hline & \multirow{2}{*}{ Examination1 } & \multirow{2}{*}{ Examination2 } & \multirow{2}{*}{ Examination 3 } & \multirow{2}{*}{ Evolution from 1 to 2 } & Evolution & Evolution \\
\cline { 4 - 7 } & & & & & & \\
from 2 to 3 & from 1 to 3 \\
\hline Averages & $-1,80$ & $-2,25$ & $-2,60$ & $-0,45$ & $-0,35$ & $-0,80$ \\
\hline Standard deviation & 1,63 & 1,65 & 1,66 & 0,48 & 0,45 & 0,54 \\
\hline
\end{tabular}

Table 4: Evolution of the Average Power of Myopia in 4 Years.

\section{Evolution of myopia between the 2nd and 4th} year

The worsening of myopia was also observed between the 2 nd and 4 th year and concerned $82 \%$ of eyes against a stability or regression at the level of $9 \%$ of eyes. The average progression of myopia between 2 and 4 years was $-0.35+/-0.45 \mathrm{D}$ with extremes of -0.25 and $-2.00 \mathrm{D}$ (Figures $1 \& 3$, Tables 3 \& 4).

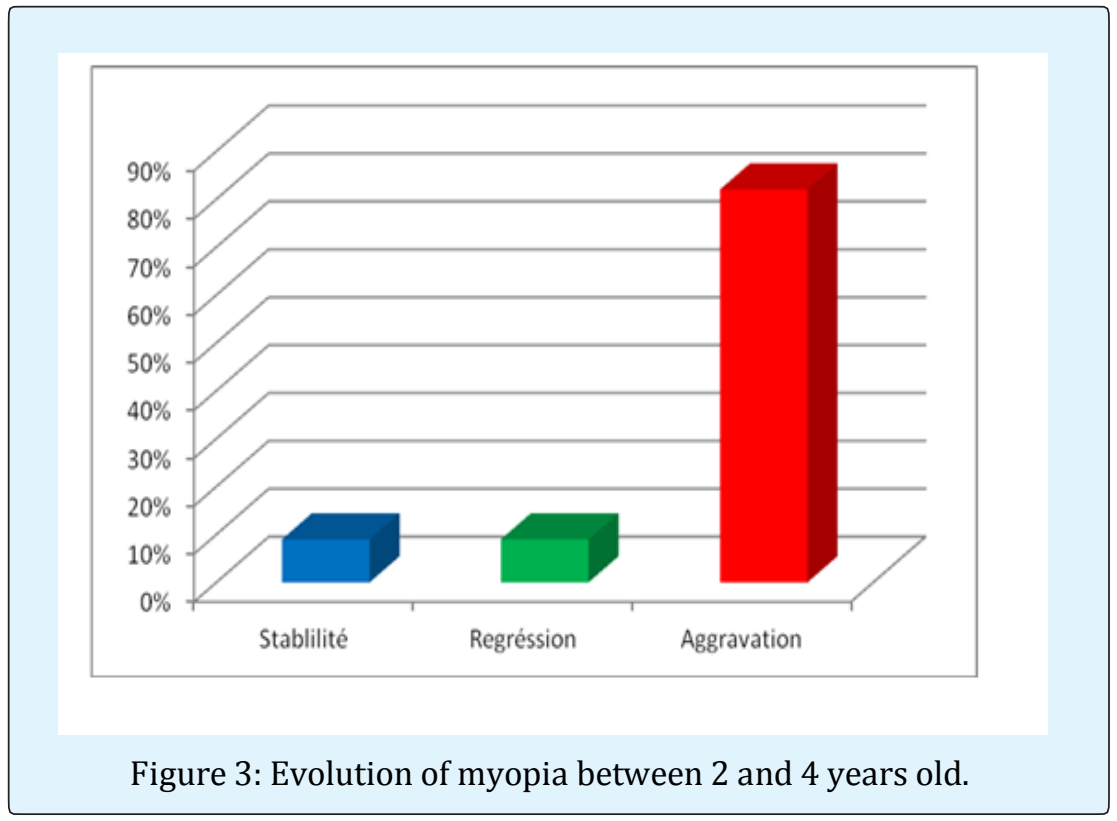

\section{Evolution of myopia in 4 years}

We observed in 4 years a worsening of myopia at the level of $93 \%$ of eyes. Stability and regression affected only
$3.5 \%$ of eyes. The mean progression of myopia was -0.80 $+/-0.54 \mathrm{D}$, with extremes of $-0.25 \mathrm{D}$ and $-2.75 \mathrm{D}$. The frequency of low myopia decreased from $80 \%$ to $70 \%$, while the proportion of medium (or moderate) myopia 


\section{Open Access Journal of Ophthalmology}

increased from $15 \%$ to $25 \%$. As for the strong myopia, it remained stable at $5 \%$. The mean power of myopia increased from $-1.80+/-1.63 \mathrm{D}$ to $-2.60+/-1.66 \mathrm{D}$ in four years (Figures $1 \& 4$, Tables $3 \& 4$ ).

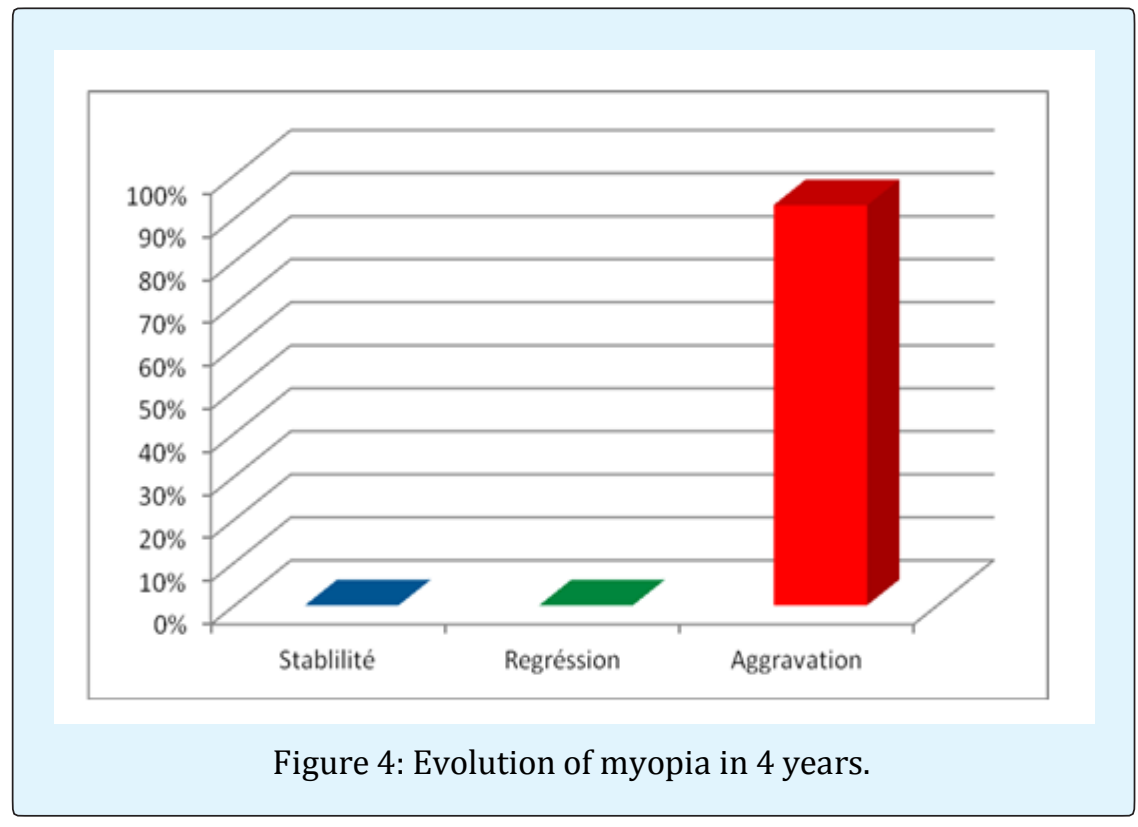

\section{Discussion}

The prevalence of nearsightedness among ametropic children in the service during the study period was $19.30 \%$. A rate well below those observed in Sweden $(50 \%)$ and South-East Asia (60-70\%) [2-4]. Sounouvou reported a prevalence of $29.4 \%$ in primary schools in Cotonou, Benin, while Nonon Saa found a prevalence of $5 \%$ among primary school children in the central region of Togo $[5,6]$. Variations in prevalence probably related to the roles of genetic and environmental factors in the occurrence of this refractive defect, but also to differences in methodology between studies.

Our study population was characterized by a feminine predominance of myopia (Table 1) which, despite recruitment bias, was similar to the feminine predominance reported by Grovesnor and Cheng in studies conducted in the United States and Canada, respectively $[7,8]$. However, our results differ from those of Zylberman who found a male-dominated predominance of myopia in a context where male children studied much longer than girls who helped with household chores [9].

We noted an average age of $8.47+/-2.08$ years with a high prevalence (78\%) of children aged 6-10 years (Table 2 ). The so-called school myopia takes root in childhood and represents $60 \%$ of myopia. It occurs between 9 and 11 years of age but can occur earlier with often high power $[10,11]$. The drop in visual acuity from afar leads to learning difficulties that most often attract the attention of school supervisors. In view of the visual handicap caused by myopia and its potentially blinding nature, early detection should be sought through systematic consultations with children's ophthalmology, combined with public awareness raising and a low cost of corrective lenses in order to improve the support of myopia from childhood onwards in Côte d' Ivoire.

Myopia is the most widespread refractive defect in the world, so WHO speaks of a myopic pandemic due to its increasing prevalence in populations [2]. In Asia, where it affects $60-80$ per cent of the population, whereas in Africa, the prevalence is estimated to be $10-20$ per cent of the population [12-16]. These differences in prevalence by race, region and time confirm the intricate roles of the innate and the acquired in the occurrence of myopia.

The prevalence of different types of myopia, according to diopter power, also differs between populations and regions. This study showed a prevalence ranging from $80 \%$ to $70 \%$ for low myopia compared to $15 \%$ to $25 \%$ for moderate and $5 \%$ to $6 \%$ for high myopia. In France, Metamoros reported prevalence of $64.19 \% ; 25.84 \%$ and 9.97\% respectively for low, moderate and high myopia in 


\section{Open Access Journal of Ophthalmology}

a subpopulation with an average age of 38.5 years [17] That is, a higher prevalence of high myopia than in our sample, which consisted of a younger population. This could be explained by the natural progression of myopia.

This defect of refraction is in fact characterized by its aggravation during growth or even beyond. Stabilization of myopia is an important issue in reducing chorioretinal complications that can lead to irreversible blindness. Methods of curbing myopia include contact lens wear, orthokeratology, atropinization, reduction of near vision time, and increased outdoor activity [18]. In this study, we observed, in children with corrective lenses, an average progression of $-0.45+/-0.48$ diopter (D) after the first two years, $-0.35+/-0.45 \mathrm{D}$ between the second and fourth year, and finally an average progression of $-0.80+/-0.54$ $\mathrm{D}$ after 4 years. The worsening affected all types of myopia (Figures 1-4). Chia reported an increase of $-1.20 \mathrm{D}$ in 2 years for children aged 6 to 12 with corrective lenses [19]. As a result, we found that myopia progressed more slowly than the results of its study. Reported progress is also lower than that reported by Liu, who in a study of the efficacy of atropinization and orthokeratology in controlling myopia progression; found an increase of $0.28 \mathrm{D}$ and $-0.34 \mathrm{D}$ per year in the orthokeratology and atropinization groups, respectively [20]. In addition, Chia found a progression of $-0.49 \mathrm{D}$ in 2 years in children who had benefited from atropine or orthokeratology [19]. In 2 years, in children with corrective lenses, we have seen progression rates that are close to or even lower than those obtained in 2 years with specific treatments inducing myopia to slow down, such as atropinization and orthokeratology, and less important than those observed by other authors with conventional corrective lenses or a placebo [21].

Indeed, Sankaridurg observed in children, Chinese myopic, 6 and 12-month averages of progression, respectively, of $0.55 \mathrm{D}+/-0.35 \mathrm{D}$ and $-0.78+/-0.50 \mathrm{D}$ in children with lenses reducing peripheral hypermetropic defocusing compared to -0.68D+/- 0.47D and -0.97D +/$0.48 \mathrm{D}$ with $-0.68 \mathrm{D}+/-0.48 \mathrm{D}$ in children. Walline, in a randomized study of 147 myopic children aged 8-11 years in a randomized study of gas-permeable rigid lenses (GLPG) versus hydrophilic soft lenses (HSH) reported a refractive progression of -1.56 +/- 0.95D with GLPG and $2.19+/-0.95 \mathrm{D}$ with HSL in three years $[22,23]$. Katz observed an average refractive progression of $-1.34 \mathrm{D}$ in 428 myopic Singaporean children aged 6 to 12 years in LRPG compared to -1.28 D in corrective lens wearers [24]. Khoo also reported an annual increase of -0.42 D for LRPG lenses and $-0.78 \mathrm{D}$ for conventional corrective lenses [25].
Other studies have confirmed this dampening effect of wearing rigid lenses with refractive progression that approximates or exceeds those observed in melanodermic children included in our study [26,27]. Comparing the impact of three types of corrective lenses on myopia, Cheng noted a 3-year increase of $-2.06 \mathrm{D}$ in the subgroup fitted with conventional corrective lenses, $-1.25 \mathrm{D}$ in the subgroup fitted with bifocal lenses, and -1.01D in children fitted with prismatic bifocal lenses [8].

The average progression of myopia in Caucasian children between 8 and 13 years of age is $-0.55 \mathrm{D}$ per year and $-0.63 \mathrm{D}$ per year among Chinese children in Hong Kong. The overall progression of myopia is $-0.82 \mathrm{D}$ per year among Asian children with a beneficial effect of time spent in outdoor activities on the evolution of myopia [9,28-30]. Contrary to these studies, we observed a smaller progression in our series. A study of risk factors for the progression of myopia should be carried out in our context with a view to preventing severe aggravation in children.

The evolution of myopia has been marked by worsening. Cases of stability or even regression between two tests were observed. The retrospective nature of our study does not allow us to give explanations, but refractive or index changes have been reported to explain some regression cases in the literature $[13,14,31]$.

\section{Conclusion}

This study showed a feminine predominance of myopia, with a higher incidence of low myopia and a low progression that averaged $-0.80+/-0.54$ diopters in 4 years. However, changes in epidemiological and evolutionary features are to be feared in view of the growing urbanization and the increasingly important and early schooling of children. Raising public awareness for the systematic screening of ametropia, particularly myopia, is essential to prevent amblyopia and blindness associated with this refractive errors.

\section{References}

1. Berthemy-Pellet S (2009) Myopia and lenses In Malet F: Contact lenses. Elsevier Masson, Paris pp: 309-359.

2. Parrajasegaram R (1999) Vision 2020 - The right to sight: from strategies for action. Am J ophthalmol 128(3): 359-360. 


\section{Open Access Journal of Ophthalmology}

3. Tano Y (2002) Pathological myopia: Where are we now?. Am J Ophthalmol 134(5): 645-660.

4. Gwiazda J, Hyman L, Hussein M (2003) A Randomized trial of progressive addition lentilles versus single vision lentilles on the progressive myopia in children. Invest Ophthalmol Vis Sci pp: 1492-500.

5. Sounouvou I, Tchabi S, Doutetien C (2008) Ametropia in primary school environment in Cotonou (Bénin). J Fr Ophtalmol 31: 771-775.

6. Nonon Saa KB, Atobian K, Banlan M (2013) Ametropia in primary school in the central area of Togo. J Fr Ophtalmol 36: 769-774.

7. Grovesnor T, Perrigin DM, Perrigin J, Maslovitz B (1987) Houston myopia control study: a randomized clinical trial part II Final report with patient care team. Am J optom physiol optol opt 64(7): 482-492.

8. Cheng D, Schmid KL, Woo GC, Drobe B (2010) Randomized trial of effect of bifocal and prismatic bifocal spectacles on myopic progression: two year results. Arch Ophthalmol 128(1): 12-19.

9. Zylberman R, Landau D, Berson D (1993) The influence of study habits on myopia in Jewish teenagers. J Pediatr Ophtalmol Strabismus 30(5): 319-322.

10. Edwards MH, Li RW, Lam CS (2002) The Hong Kong on the progressive control of lens myopia. Invest Ophthalmol Vis Sci 43: 2852-2858.

11. Tan NW, Saw SM, Lam DS, Cheng HM, Rajan U, et al. (2000) Temporal variations in myopia progression in Singaporean children within an academic year. Optom Vis Sci 77(9): 465-472.

12. (2006) Ethnically-specific prevalence rates of refractive errors vary among Asian Children in Malaysia and Singapore. Frère J Ophthalmol 90: 12301235.

13. Saw SM, Shankar A, Tan SB (2006) A cohort study of incidental Mypoïa in Singaporean Children. Invest Ophthalmol Vis Sci 47: 1839-1844.

14. Mondon H, Metge P (1994) Epidemiologie pathophysiologie. In strong myopia. Mondon $\mathrm{H}$, Metge P. Report of French Society of Ophtalmology. Masson éd Paris pp: 25-8.
15. Amedome KM, Ayena KD, Alaglo KA (2013) Prevalence of ametropia in School environment in Southern Togo: Case of the prefecture of the Lakes. J Rech Sci Univ Bénin (Togo) 15: 443-451.

16. Mashige KP, Jaggemath J, Ramson P, Martin C, Chinanayi FS, et al. (2016) Prevalence of refractive errors in the INK Area, Durban, South Africa. Optom Vis Sci 93: 243-250.

17. Metamoros E, Ingrand $P$, Pelen $F$, Bentaleb $Y$, Weber M, et al. (2015) Prevalence of myopia in France: A cross-sectional analysis. Medecine 94(45): e1976.

18. Walline JJ (2016) Myopia control: A Review. Eye contact lens 42(1): 3-8.

19. Chia A, Chua WH, Cheung YB (2012) Atropine for the treatment of infantile myopia: safety efficacy de $0,5 \%$, $0,1 \%$ et $0,01 \%$ of atropine for the treatment myopia. Ophtalmology 119: 347-54.

20. Yen MY, Liu JH, Kao SC, Shiao CH (1989) Comparison of the effets of the atropine and cyclopentolate myopia. Ann Ophthalmol 21(5):180-182.

21. Siatkowski RM, Cotter SA, Crockett RS (2008) Twoyear multicenter, randomized, double-masked, placebo-controlled, parallel safety and efficacy study of $2 \%$ pirenzépine ophthalmic gel in children with myopia. JAAPOS 12: 332-339.

22. Padmaja Sankaridurg, Brien Holden, Earl Smith, Thomas Naduvilath, Xiang Chen, et al. (2011) Decrease in rate of myopia progression with a contact lens desingned to reduce relative peripheral hyperopia: one-year results. Invest Ophthalmol Vis Sci 52(13): 9362-9367.

23. Walline JJ, Gaume A, Jones LA, Rah MJ, Manny RE, et al. (2007) Benefits of contact lens wear for children and teens. Eye Contact Lens 33(6 Pt 1): 317-321.

24. Katz J, Schein OD, Levy B, Cruiscullo T, Saw SM, et al. (2003) A randomized trial of rigid gas permeable contact lenses to reduce progression of children's myopia. Am J Ophthalmol 136(1): 82-90.

25. Khoo CY, Chong J, Rajan U (1999) A 3-year study on the effect of RGP contact lenses on myopic children. Singapore Med J 40(4): 230-237.

26. Horner DG, Soni PS, Salmon TO, Swartz TS (1999) Myopia progression in adolescent wearers of soft 


\section{Open Access Journal of Ophthalmology}

contact lenses and spectacles. Optom Vis Sci 76(7): 474-479.

27. Perrigin J, Perrigin D, Quintero S, Grosvenor T (1990) Silicone-acrylate contact lenses for myopia control: 3year results. Optom Vis Sci 67(10): 764-769.

28. Fan DS, Lam DS, Lam RF, Lau JT, Chong KS, et al. (2004) Prevalence, incidence, and progression of myopia of school children in Hong Kong. Invest Ophthalmol Vis Sci 45(4): 1071-1075.

29. Donovan L, Sankaridurg P, Ho A, Naduvilath T, Smith EL, et al. (2012) Myopia progression rates in urban children wearing single-vision spectacles. Optom Vis Sci 89(1): 27-32.

30. Guggenheim JA, Northstone K, McMahon G, Ness AR, Deere K, et al. (2012) Time outdoors and physical activity as predictors of incident myopia in childhood: a prospective cohort study. Invest Ophthalmol Vis Sci 53(6): 2856-2865.

31. Cho P, Cheung SW (2012) Retardation of Myopia in Orthokeratology (ROMIO) Study: A 2-year randomized clinical trial. Invest Ophthalmoi vis Sci 53(11): 7077-7085.

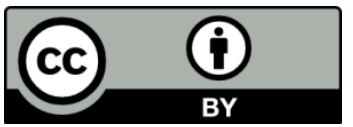

